Review

\title{
Pathogenesis of Lassa Fever
}

\section{Nadezhda E. Yun and David H. Walker*}

Department of Pathology, University of Texas Medical Branch, Galveston, Texas, USA;

E-Mails: neyun@utmb.edu (N.Y.); dwalker@utmb.edu (D.W.)

* Author to whom correspondence should be addressed; E-Mail: dwalker@utmb.edu (D.W.); Tel.: +1-409-772-3989; Fax: +1-409-772-1850.

Received: 3 September 2012; in revised form: 28 September 2012 / Accepted: 3 October 2012 / Published: 9 October 2012

\begin{abstract}
Lassa virus, an Old World arenavirus (family Arenaviridae), is the etiological agent of Lassa fever, a severe human disease that is reported in more than 100,000 patients annually in the endemic regions of West Africa with mortality rates for hospitalized patients varying between $5-10 \%$. Currently, there are no approved vaccines against Lassa fever for use in humans. Here, we review the published literature on the life cycle of Lassa virus with the specific focus put on Lassa fever pathogenesis in humans and relevant animal models. Advancing knowledge significantly improves our understanding of Lassa virus biology, as well as of the mechanisms that allow the virus to evade the host's immune system. However, further investigations are required in order to design improved diagnostic tools, an effective vaccine, and therapeutic agents.
\end{abstract}

Keywords: arenavirus; Lassa virus; pathogenesis; cell-mediated immunity 


\section{Phylogeny and geographic distribution}

Lassa virus, the causative agent of Lassa fever, belongs to the family Arenaviridae. Arenaviruses are classified as segmented negative-sense RNA (nsRNA) viruses and are phylogenetically closely related to other segmented nsRNA viruses belonging to Bunyaviridae and Orthomyxoviridae. The three virus families share basic features of the intracellular replication cycle. Based on serological cross-reactivity [1], phylogenetic relations [2], and geographical distribution, all arenaviruses are further sub-divided into the Old World and New World virus complexes. The New World arenavirus complex comprises viruses that circulate in North America (i.e., Whitewater Arroyo (WWAV), Tamiami (TAMV), and Bear Canyon (BCNV) viruses) and South America (i.e., Tacaribe (TACV), Junin (JUNV), Machupo (MACV), Guanarito (GTOV), and Sabia (SABV) viruses). The Old World complex includes arenaviruses that circulate in Africa, Europe, and Asia (i.e., lymphocytic choreomeningitis (LCMV) and Lassa (LASV) viruses). The range of reservoir rodent species restricts the geographic occurrence of arenaviruses, with the exception of LCMV that is distributed worldwide owing to its association with Mus domesticus and M. musculus, which have migrated globally (Table 1). TACV is the only arenavirus that has been isolated from fruit-eating bats. The reservoir hosts of LASV are rodents of the genus Mastomys that are enzootic in sub-Saharan Africa [3]. In LASV endemic regions, up to $30 \%$ of Mastomys rodents can carry the virus [4]. There is strong phylogenetic evidence supporting the hypothesis that the diversity of arenaviruses resulted from a long-term coevolutionary relationship with rodents of the family Muridae [2,5]. At least seven arenaviruses are known to cause severe hemorrhagic fever in humans, among which are LASV, JUNV, MACV, GTOV, and SABV that are endemic in West Africa, Argentina, Bolivia, Venezuela, and Brazil, respectively [6], and recently discovered Lujo (LUJV) and Chapare (CHAPV) viruses that originated in Zambia and Bolivia, respectively [7,8]. These viruses, except SABV, LUJV, and CHAPV are included in the NIAID's Category A Priority Pathogens list, and all experimental work with these agents is only permitted in Biosafety Level 4 (BSL-4) facilities.

\section{Virion structure, genome organization and expression}

Arenaviruses have pleomorphic virions from 40 to more than $200 \mathrm{~nm}$ in diameter that consist of nucleocapsid surrounded by a lipid envelope [10]. On electron micrographs the interior of virions shows a characteristic granular appearance due to incorporation of host cell ribosomes in virus particles during assembly. This, yet unexplained, phenomenon was the basis for the family name ( arenosus $=$ sandy). The genome of arenaviruses consists of two single-stranded RNA segments, small (S) and large (L). Both genomic segments have an ambisense gene organization and encode two genes in opposite orientation. The L RNA $(\sim 7 \mathrm{~kb})$ encodes the viral RNA-dependent RNA polymerase $(\mathrm{L})$ and the small RING finger zinc-binding protein $(Z)$. The S RNA $(\sim 3.4 \mathrm{~kb})$ encodes the glycoprotein precursor protein (GPC) and the nucleoprotein (NP). GPC is posttranslationally cleaved to yield two envelope glycoproteins GP1 and GP2 and the stable signal peptide (SSP). 
Table 1. Arenaviridae family (adapted from reference [9]).

\begin{tabular}{|c|c|c|c|c|c|}
\hline Virus & Acronym & Distribution & Reservoirs & $\begin{array}{l}\text { Human } \\
\text { Pathogen }\end{array}$ & $\begin{array}{l}\text { Mortality } \\
\text { Rate }\end{array}$ \\
\hline \multicolumn{6}{|c|}{ Old World arenaviruses } \\
\hline Ippy & IPPYV & $\begin{array}{l}\text { Central African } \\
\text { Republic }\end{array}$ & Arvicanthis sp. & \multicolumn{2}{|l|}{ not reported } \\
\hline Lassa & LASV & West Africa & Mastomys sp. & Yes & High \\
\hline $\begin{array}{l}\text { Lymphocytic } \\
\text { choriomeningitis }\end{array}$ & LCMV & Europe, Americas & Mus musculus & Yes & Low \\
\hline Mobala & MOBV & $\begin{array}{l}\text { Central African } \\
\text { Republic }\end{array}$ & Praomys sp. & \multicolumn{2}{|l|}{ not reported } \\
\hline Mopeia & MOPV & $\begin{array}{l}\text { Mozambique, } \\
\text { Zimbabwe }\end{array}$ & Mastomys natalensis & \multicolumn{2}{|l|}{ not reported } \\
\hline \multicolumn{6}{|c|}{ New World arenaviruses } \\
\hline Allpahuayo & ALLV & Peru & $\begin{array}{l}\text { Oecomys bicolor, } \\
\text { Oecomys paricola }\end{array}$ & \multicolumn{2}{|l|}{ not reported } \\
\hline Amapari & AMAV & Brazil & $\begin{array}{l}\text { Oryzomys capito, } \\
\text { Neacomys guianae }\end{array}$ & \multicolumn{2}{|l|}{ not reported } \\
\hline Bear Canyon & $\mathrm{BCNV}$ & USA & Peromyscus californicus & \multicolumn{2}{|l|}{ not reported } \\
\hline Chapare & CHPV & Bolivia & unknown & Yes & unknown \\
\hline Cupixi & CPXV & Brazil & Oryzomys sp. & \multicolumn{2}{|l|}{ not reported } \\
\hline Flexal & FLEV & Brazil & Oryzomys spp. & Yes & non-fatal \\
\hline Guanarito & GTOV & Venezuela & Zygodontomys brevicauda & Yes & High \\
\hline Junin & JUNV & Argentina & Calomys musculinus & Yes & High \\
\hline Latino & LATV & Bolivia & Calomys callosus & \multicolumn{2}{|l|}{ not reported } \\
\hline Machupo & MACV & Bolivia & Calomys callosus & Yes & High \\
\hline Oliveros & OLVV & Argentina & Bolomys obscurus & \multicolumn{2}{|l|}{ not reported } \\
\hline Parana & PARV & Paraguay & Oryzomys buccinatus & \multicolumn{2}{|l|}{ not reported } \\
\hline Pichinde & PICV & Colombia & Oryzomys albigularis & \multicolumn{2}{|l|}{ not reported } \\
\hline Pirital & PIRV & Venezuela & Sigmodon alstoni & \multicolumn{2}{|l|}{ not reported } \\
\hline Sabia & SABV & Brazil & unknown & Yes & unknown \\
\hline Tacaribe & TACV & Trinidad & Artibeus spp. & Yes & non-fatal \\
\hline Tamiami & TAMV & USA & Sigmodon hispidus & \multicolumn{2}{|l|}{ not reported } \\
\hline Whitewater Arroyo & WWAV & USA & Neotoma albigula & Yes & Low \\
\hline \multicolumn{6}{|c|}{ Status remains pending } \\
\hline Lujo & LUJV & $\begin{array}{l}\text { Zambia, South } \\
\text { Africa }\end{array}$ & unknown & Yes & High \\
\hline Luna & $\overline{\text { LUNV }}$ & Zambia & Mastomys natalensis & \multicolumn{2}{|l|}{ not reported } \\
\hline Lunk & LNKV & Zambia & Mus minutoides & \multicolumn{2}{|l|}{ not reported } \\
\hline Kodoko & KODV & Africa & Mus minutoides & \multicolumn{2}{|l|}{ not reported } \\
\hline Dandenong & DANV & unknown & unknown & \multicolumn{2}{|l|}{ not reported } \\
\hline Merino Walk & MWV & South Africa & Myotomys unisulcatus & \multicolumn{2}{|l|}{ not reported } \\
\hline
\end{tabular}

The enzymatic machinery for RNA synthesis in arenaviruses is contained within a single L polymerase protein. This $250-450 \mathrm{kDa}$ protein utilizes viral RNA templates that consist of genomic RNA encapsidated by the viral nucleocapsid protein NP and comprises viral ribonucloprotein (RNP) [10]. L polymerase of arenaviruses contains the SDD motif characteristic of all RNA-dependent RNA polymerases (RdRp). Upon infection, once the virus RNP is delivered into the cytoplasm of the host cell, the L polymerase associated with the viral RNP initiates transcription from the genome 
promoter located at the 3'-end of each genomic RNA segment, L and S. The 5' and 3' terminal 19 nt viral promoter regions of both RNA segments required for the recognition and binding by the viral polymerase [11] exhibit a high degree of conservation among arenaviruses. The genome segments have highly complementary 5'- and 3'-ends (19 nt) that have been predicted to form panhandle structures [12]. The primary transcription results in the synthesis of mRNA of viral genes encoded in antigenomic orientation, NP and L polymerase, from the S and L segments, respectively. Transcription terminates at the distal side of the stem-loop (SL) structure within the intergenomic region (IGR). This SL structure has been proposed to stabilize the 3'-termini of the viral mRNAs [13,14]. Arenaviruses utilize a cap snatching strategy to acquire the cap structures of cellular mRNAs. Cap snatching is mediated by the endonuclease activity of the $\mathrm{L}$ polymerase that is co-factored by the cap binding activity of NP [15-17]. Therefore, arenaviruses produce capped non-polyadenylated mRNAs. Subsequently, the L polymerase adopts a replicase mode and moves across the IGR to generate a fulllength complementary antigenomic RNA (agRNA). This agRNA serves as a template for the synthesis of mRNAs of viral genes encoded in genomic orientation, GPC and Z, from the S and L segments, respectively, and for the synthesis of full-length genomic RNA (gRNA) [10]. Both gRNA and agRNA of arenaviruses contain a nontemplate $G$ residue at their 5'-ends [18]. The proposed "prime and realign" mechanism includes the synthesis of a $\mathrm{pppG}_{\mathrm{P}} \mathrm{C}_{\mathrm{OH}}$ dinucleotide primer from the CG nucleotides at positions +2 and +3 of the 3 '-end genome promoter sequence, that is then realigned such that its 3'-terminal $\mathrm{C}_{\mathrm{OH}}$ is opposite the genome 3'-terminal $\mathrm{G}$ residue, and the realigned $\mathrm{pppG}_{\mathrm{P}} \mathrm{C}_{\mathrm{OH}}$ then acts as a primer for a complementary RNA strand synthesis [19, 20]. The matrix protein $Z$ is not required for viral genome transcription and replication; however, $Z$ exhibits a dose-dependent inhibitory effect on viral RNA synthesis [21-23]. This inhibitory effect of Z has been reported for New World [24], as well as for Old World [25] arenaviruses.

In addition to the functions required to support virus replication, at least two viral proteins, NP and $\mathrm{Z}$, have been proposed to modulate the host cell response to infection. NP is the most abundant viral protein both in infected cells and in virions, comprises the main structural component of the viral ribonucleoprotein (RNP) and plays an essential role in the synthesis of viral RNA [10]. Recent experimental data indicate that NP is involved in virus-induced inhibition of type I IFN signaling [26-28]. This activity has been mapped to the C-terminal domain of NP, which has a folding that mimics that of the DEDDH family of 3'-5' exoribonucleases [29]. Functional analysis confirmed the exonuclease activity of LASV NP that has been proposed to be critical for its type I IFN counteracting function [15]. The small RING finger protein $\mathrm{Z}$ is the arenavirus counterpart of the matrix (M) protein of other negative sense RNA viruses [10]. Z protein of LCMV interacts with the promyelocytic leukemia (PML) protein and the eukaryotic translation initiation factor 4E (eIF4E) in infected cells and has been proposed to contribute to the noncytopathic nature of LCMV infection and repression of capdependent translation [30-33].

\section{Epidemiology of Lassa fever}

LASV was first isolated in 1969 from a missionary nurse who worked in a clinic in a small town, Lassa, in northeastern Nigeria [34]. The nurse presumably acquired infection from an obstetrical patient residing in Lassa. She died approximately one week after the onset of symptoms. Subsequently, 
two more nurses that attended the first patient contracted the disease, which was later named Lassa fever and caused the death of one of them. Infectious virus was isolated from all three cases [35].

Initially, several countries of West Africa were identified to be endemic for LASV, namely Sierra Leone [4,36], Guinea [37,38], Liberia [39-41], and Nigeria [42-45]. However, a serological survey among patients admitted with a history of fever and missionaries that had experienced a febrile illness showed that LASV was also present in Ivory Coast, Mali, and Central African Republic [46]. The notion that LASV was endemic in larger areas of West Africa was further supported by the results of investigation of an imported case of Lassa fever in Germany in 2000. During the incubation period, the index patient traveled through several countries, namely Ghana, Ivory Coast, and Burkina Faso, that were not considered to be endemic at that time [47]. Later, cases of Lassa fever have been reported from Burkina Faso, Ivory Coast, Ghana, Senegal, Gambia, and Mali [48,49].

According to estimations, LASV is responsible for 100,000-300,000 infections and approximately 5,000 deaths annually [50]. However, the high degree of seroprevalence of LASV-specific antibodies in the general population residing in the endemic regions, although highly variable depending on the geographical location (from $1.8 \%$ to $55 \%$ ) [4,37,43,51], indicates that most infections are mild or possibly even asymptomatic and do not result in hospitalization. This is also supported by the findings indicating a high incidence of LASV-specific seroconversion, from $5 \%$ to $20 \%$ of the nonimmune population per year [4]. Nosocomial outbreaks are associated with higher mortality rates ranging from $36 \%$ to $65 \%$ [40,42,52]. However, serosurveillance studies in hospitals dealing with suspected Lassa fever cases showed that the hospital staff that routinely practiced basic hygiene measures had no higher risk of infection than the local population [53]. Infection with LASV presumably occurs through contact with body fluids or excreta, or inhalation of aerosols produced by infected animals. LASV is stable in aerosol [54], and animal-to-animal transmission via the airborne route has been demonstrated in the laboratory setting [55]. Hunting of peridomestic rodents and consumption of their meat is another important route of LASV transmission to humans residing in endemic areas of West Africa [38].

The multimammate mouse, Mastomys natalensis, was originally identified as the primary host species for LASV [56]. However, due to the poor understanding of the taxonomy of the genus, it is uncertain which species and particular subspecies serve as a reservoir for the virus [3]. The studies addressing the importance of $M$. natalensis for the circulation of LASV in nature demonstrated that newborn animals inoculated intraperitoneally develop persistent asymptomatic infection [57]. Significant infectious virus titers were detected in many organs, tissues, and fluids including lymph node, liver, spleen, lung, blood, and brain up to 74 days after inoculation. Moreover, LASV was isolated from the urine and throat swabs of infected animals. No significant histopathological alterations were observed in these animals. Interestingly, adult M. natalensis infected with LASV also developed a disseminated infection that lasted up to 30 days. Some animals cleared the virus from some organs, but there was persistence in other organs up to 103 days when the study was terminated. The only consistent histopathological finding observed in adult animals was a moderate chronic meningoencephalitis [57]. These data demonstrate that $M$. natalensis has an optimal pattern of infection and virus shedding for the maintenance of LASV in nature. 


\section{Clinical description and pathogenesis of Lassa fever}

The incubation period of Lassa fever ranges from 7 to 21 days [34,58]. The clinical disease begins as a flu-like illness characterized by fever, general weakness, and malaise, which may be accompanied by cough, sore throat, and severe headache. Gastrointestinal manifestations such as nausea, vomiting, and diarrhea are also common (Table 2). The differential diagnosis of Lassa fever based on the presenting symptoms can be problematic due to the many other acute undifferentiated febrile illnesses circulating in West Africa [58,59]. Although, hemorrhagic manifestations are not an important feature of Lassa fever, perturbation of vascular function is likely to be central to Lassa fever-associated pathobiology, since the signs of increased vascular permeability, such as facial edema and pleural and pericardial effusions, indicate a poor prognosis for the disease outcome. Recovery from Lassa fever generally begins within 8 to 10 days of disease onset. In severe cases, the condition of the patient deteriorates rapidly between the 6th and 10th day of illness with severe pulmonary edema, acute respiratory distress, clinical signs of encephalopathy, sometimes with coma and seizures, and terminal shock. Bleeding from mucosal surfaces is often observed; however, it is usually not of a magnitude to produce shock by itself [58]. Sensorineural deafness is commonly observed in patients in the late stages of disease or in early convalescence in survivors [60].

The level of viremia is highly predictive of the disease outcome. In a study involving 137 patients with Lassa fever, patients that presented with viremia $<10^{3}$ median tissue culture infectious dose $\left(\mathrm{TCID}_{50}\right) / \mathrm{ml}$ on the day of hospitalization had 3.7 times greater chance of survival than those admitted with higher levels of viremia. Similarly, the probability of fatal outcome in patients with serum titers $>$ $10^{3} \mathrm{TCID}_{50} / \mathrm{ml}$ and serum levels of aspartate aminotransferase (AST) $\geq 150$ international units (IU)/L was 21 times higher than that in patients not meeting either of these criteria. Virtually all patients with fatal Lassa fever whose sera were tested were viremic at the time of death with terminal viremia ranging from $10^{3}$ to $10^{8} \mathrm{TCID}_{50} / \mathrm{ml}$ [61]. Detailed studies have shown that viremia peaks between 4 and 9 days after the onset of symptomatic disease and is followed by pronounced clinical manifestations. Patients recovering from Lassa fever clear virus from blood circulation about 3 weeks after the beginning of illness [40,61-63].

The current knowledge of Lassa fever pathogenesis does not include the chain of events that take place during disease development and lead to death of severely ill patients. Apparently, failure to develop the cellular immune response that would control dissemination of LASV, which is indicated by high serum virus titers, combined with disseminated replication in tissues and absence of neutralizing antibodies, leads to the development of fatal Lassa fever [64]. However, considering the high mortality and truly dramatic course of the disease, the pathological findings do not provide the basis that would explain the mechanism of disease progression and the cause of death from Lassa fever. 
Table 2. Onset and duration of the principal clinical manifestations of Lassa fever (adapted from reference [58]).

\begin{tabular}{rrrr}
\hline Clinical signs & \multicolumn{2}{c}{ Day of illness } & $\begin{array}{r}\text { Duration, } \\
\text { and symptoms }\end{array}$ \\
\cline { 2 - 3 } Start day & End day & 10 \\
Fever & 1 & 11 & 11 \\
Weakness & 3 & 14 & 11 \\
Cough & 3 & 13 & 9 \\
Chest pain & 4 & 12 & 8 \\
Back pain & 4 & 12 & 8 \\
Joint pain & 4 & 11 & 7 \\
Sore throat & 4 & 10 & 6 \\
Dysuria & 4 & 11 & 7 \\
Headache & 4 & 8 & 3 \\
Abdominal pain & 5 & 9 & 4 \\
Vomiting & 5 & 9 & 4 \\
Diarrhea & 5 & 12 & 5 \\
Pharyngitis & 7 & 12 & 5 \\
Conjunctivitis & 7 & 11 & 4 \\
Bleeding & 7 & 14 & 5 \\
Abdominal & 9 & 14 & 5 \\
Rales & 9 & 16 & 7 \\
Facial edema & 9 & &
\end{tabular}

Physical examination of patients after the onset of fever often reveals purulent pharyngitis, bilateral conjunctival hemorrhages, facial edema, and generalized abdominal tenderness. Macroscopic pathological changes can include pleural effusions, pulmonary edema, ascites, and hemorrhagic manifestations in the gastrointestinal mucosa [34,65]. Microscopic findings include hepatocellular necrosis and apoptosis, splenic necrosis, adrenocortical necrosis, mild mononuclear interstitial myocarditis without myocardial fiber necrosis, alveolar edema with capillary congestion and mild interstitial pneumonitis, lymph nodal sinus histiocytosis with mitoses, gastrointestinal mucosal petechiae, renal tubular injury, and interstitial nephritis $[34,59,66]$. A comprehensive postmortem histopathological examination of 21 virologically confirmed community-acquired cases of Lassa fever in Sierra Leone revealed [59] variable levels of hepatic necrosis involving from 1 to $40 \%$ of hepatocytes. The necrotic hepatocytes were randomly distributed often forming foci of contiguous cells. Mononuclear phagocytes were observed either contacting or phagocytosing necrotic hepatocytes. Interestingly, this phagocytic reaction, although highly variable from case to case and even from one necrotic focus to another in the same case, demonstrated a tendency towards homogeneity of the level of involvement within a particular patient. The predominant distribution of splenic necrosis was observed in the marginal zone of the periarteriolar lymphocytic sheath. Close examination of thin tissue sections revealed the presence of fibrin in addition to the debris of necrotic cells. Splenic venous subendothelium appeared to be infiltrated by lymphocytes and other mononuclear cells. Microscopic examination of adrenal glands showed prominent spherical, hyaline, acidophilic cytoplasmic inclusions in cells near the junction of zona reticularis and medulla. In most cases these cells appeared to be adrenocortical cells of the zona reticularis; however, some cells were of adrenal medulla origin. 
Additionally, multifocal adrenocortical cellular necrosis was detected that was most prominent in the zona fasciculata and was often associated with focal inflammatory reaction. However, in all examined cases adrenal necrosis was mild and $\geq 90 \%$ of the cells of adrenal cortex appeared viable [59].

The major and most common lesions of Lassa fever in humans occur in the liver $[34,59,66,67]$. There are four principal features of LASV hepatitis can be derived: 1) focal cytoplasmic degeneration of hepatocytes suggestive of phagocytosed apoptotic fragments; 2) randomly distributed multifocal hepatocellular necrosis; 3) monocytic reaction to necrotic hepatocytes; 4) hepatocellular mitoses. These morphologic effects do not uniformly occur in all cases, but in some instances can be found simultaneously $[59,66]$. Based on the degree of hepatic damage, three general nosopoetic phases have been proposed to divide patients with fatal Lassa fever into categories with respect to pathogenic events in fatal LASV hepatitis [66]. The first phase, active hepatocellular injury, is defined by the presence of focal cytoplasmic degeneration with $<20 \%$ of hepatocytes undergoing necrosis. This phase may represent the late stage of viremic spread and early cellular injury, which is, most likely, caused by direct viral action rather than mediated by a cellular immune response, since lymphocytic infiltration is not detected. The second phase, the peak of Lassa hepatitis, is characterized by $20-50 \%$ necrosis of hepatocytes, widespread focal cytoplasmic degeneration and limited phagocytic infiltration. This is suggested not only progressive hepatocellular damage, but, also, early liver recovery through phagocytic removal of necrotic hepatocytes and regeneration of new cells. The third phase, hepatic recovery, is defined by $<10 \%$ of hepatocellular necrosis, absence of focal cytoplasmic degeneration and clear evidence of mitoses, which indicate liver regeneration [66]. Interestingly, no correlation has been observed between the degree of hepatic necrosis and chemical indicators of liver damage, such as elevated levels of AST, alanine transaminase (ALT), and lactate dehydrogenase (LDH) in serum [59, 66]. Overall it is apparent that the level of liver damage can vary dramatically in patients that die from Lassa fever. Therefore, it can be concluded that liver disease is a necessary, but not sufficient, condition in the chain of pathological events that lead to fatal outcome.

Lassa fever is not considered to be associated with coagulation dysfunction, e.g., neither decrease in the coagulation factors nor disseminated intravascular coagulation (DIC) has been observed in infected patients. However, moderate thrombocytopenia with significantly impaired functionality of thrombocytes is detected in patients with severe Lassa fever [68].

One possible mechanism involved in Lassa fever pathogenesis could be infection-triggered induction of uncontrolled cytokine expression similar to what is seen in sepsis. This hypothesis is supported by experimental data obtained from a case of fatal Lassa fever imported into Germany in 2000 [69]. In this patient, who died from multi-organ failure and hemorrhagic shock, the proinflammatory cytokines, interferon $\gamma$ (IFN- $\gamma$ ) and tumor necrosis factor $\alpha$ (TNF- $\alpha$ ), rose to extremely high levels shortly before death. However, in another study no elevation of both cytokine levels was observed in the examined fatal cases of Lassa fever, which suggests that the levels of IFN- $\gamma$ and TNF- $\alpha$ are either elevated only in a fraction of patients or during a very short period that would require frequent sampling for detection [70].

Another possibility is that virus-induced immunosuppression may be involved in the pathogenesis of severe Lassa fever [71]. Thus, infection with LASV fails to activate monocyte-derived dendritic cells (DC) and macrophages (MP) of human origin. Infected DC fail to secrete proinflammatory cytokines, do not upregulate costimulatory molecules, such as CD40, CD80, and CD86, and poorly 
induce proliferation of $\mathrm{T}$ cells [72,73]. Importantly, human $\mathrm{DC}$ infected with the naturally nonpathogenic Mopeia virus, a closely related arenavirus that shares $75 \%$ amino acid similarity with LASV and was isolated from the same rodent reservoir [2], induces stronger CD4 and CD8 T-cell responses than those infected with LASV [74]. Downregulation of immune responses caused by LASV infection demonstrated in vitro is also in agreement with the results of clinical observations showing that fatal outcome of Lassa fever correlates with low levels or absence of interleukin (IL) 8 and IFN inducible protein 10 (IP-10) in circulation [70].

\section{Immune responses to LASV infection}

Patients infected with LASV produce $\operatorname{IgM}$ and $\operatorname{IgG}$ antibody isotypes [61]. However, since both immunoglobulin classes are detected in viremic patients, most likely the antibodies that are produced early in infection are not neutralizing. This is in agreement with reports showing that neutralizing antibodies appear months after acute infection is resolved, and the titers are often low [75]. Interestingly, the neutralizing antibody titers continue to rise even several months after convalescence has been established, which may indicate constant stimulation of B cells due to low levels of virus persistence. Antibodies in seroconverted individuals are specific to GPC, NP, and, likely, Z protein [76-79]. Experiments aimed at the identification of B-cell antigenic epitopes elucidated four sites on NP, two sites on GP1, and six sites on GP2 [80-82]. Some antibodies are highly LASV strainspecific, while other react with a broad range of arenaviruses including African and South American members of the family [80,81].

Since neutralizing antibodies appear in survivors long after virus has been cleared, it has been considered that resolution of LASV infection is mediated by cellular immunity. This hypothesis is supported by experimental data showing that cynomolgus monkeys that survive LASV infection have high concentrations of activated $\mathrm{T}$ lymphocytes and efficiently control viral replication. In contrast, the animals that succumb to infection display delayed, low-level T-cell activation, uncontrolled viral replication, and develop fatal disease [83]. Seropositive individuals residing in endemic areas have very strong memory CD4+ T-cell responses, and the antigenic epitopes are mapped to NP and a 13 amino acid region in the N-terminal part of GP2 that is highly conserved between Old and New World arenaviruses $[84,85]$. Importantly, a recent study showed that transgenic mice that express human major histocompatibility complex class I (MHC-I) molecules fail to control virus replication and are susceptible to Lassa virus, which is in contrast to normal laboratory mice that rapidly clear virus. Intriguingly, CD8 and CD4 $\mathrm{T}$ cell depletion in these mice prevents disease despite high levels of viremia [64]. These data indicate that $\mathrm{T}$ cells are essential for rapid resolution of LASV infection; however, if the host fails to control virus replication due to inadequate activation of the immune system, T lymphocytes may play a key role in Lassa fever pathogenesis.

\section{Animal models of Lassa fever}

Mice and guinea pigs have been evaluated as models of LASV infection [55]. However, normal adult mice are highly resistant to peripheral routes of inoculation. Mice expressing humanized MHC-I are LASV-susceptible and develop a severe illness [64]. Genotype of a particular mouse strain has significant influence on the development of pathologic manifestations in infected mice. Thus, newborn 
mice develop an asymptomatic infection upon inoculation with LASV with high virus titers in the brain, lung, and muscle, while intracranial inoculation of adult mice results in a fatal convulsive disorder resembling classical murine LCM immunopathology. However, other investigators failed to reproduce these findings $[55,86,87]$. Pathogenicity of LASV for guinea pigs largely depends on the host strain and the virus used for inoculation. For instance, Josiah strain of LASV causes a uniformly lethal disease in inbred strain 13 guinea pigs; however, the lethality varies from approximately $30 \%$ to more than $60 \%$ in outbred Hartley guinea pigs. Inbred animals have higher viral titers in all target tissues than outbred guinea pigs [57,88]. LASV-infected guinea pigs destined to die develop respiratory insufficiency with pulmonary edema, alveolar hyaline membranes, myocarditis, and focal calcification of myocardial fibers and hepatocytes. Terminally ill animals are viremic and contain virus in nearly every organ tested. There are two major differences between Lassa fever pathogenesis in humans and guinea pigs. In humans LASV is particularly hepatotropic, and patients with severe Lassa fever develop hepatocellular necrosis; however, in guinea pigs only foci of calcified hepatocytes are observed. On the other hand, LASV is myocardiotropic and myocardiopathic in guinea pigs, findings that have not been reported in humans [57]. Also, several isolates of LASV from clinical human cases are benign in guinea pigs, and some are lethal when tested in cynomolgus monkeys [89]. Therefore, in order to reliably model the pathogenesis of Lassa fever in humans, studies in non-human primates are required.

Several non-human primate species have been evaluated as potential models for Lassa fever including squirrel monkeys, capuchin monkeys, marmosets, hamadryas baboons, African green monkeys, cynomolgus monkeys, and rhesus monkeys [55,57,90-95]. Capuchin and squirrel monkeys seroconverted upon infection with LASV; however, the animals of both species uniformly survived low and high dose inoculations [55,57]. Virus was detected in virtually all organs of infected squirrel monkeys, but the liver, lymph nodes, and kidneys were the key early targets. Later in infection, the spleen, heart, and brain also showed pathological alterations. Viremia persisted in all animals for up to 28 days after infection. Histopathologic changes were mild and included germinal center necrosis in the spleen and lymph nodes, myocarditis, acute arteritis, renal tubular necrosis and regeneration, chronic inflammation of the choroid plexus, ependyma, and meninges, and cerebral perivascular cuffing. Hepatocytic regeneration suggesting recovery from disease-mediated liver damage was also observed [91]. Hamadryas baboons infected with LASV develop a disease that clinically resembled a severe form of human Lassa fever. The animals developed fever, characteristic pathologic and hemorrhagic manifestations, and high levels of viremia [93]. Interestingly, African green monkeys and rhesus macaques challenged with low doses (10-15 pfu) of LASV uniformly succumbed to the infection, whereas a high challenge dose $\left(10^{6} \mathrm{pfu}\right)$ was only partially lethal in these animals [55]. The gross pathological changes in LASV-infected rhesus macaques were petechiae and, in some animals, mild-to-moderate pleural effusions. Viremia appeared between days 5 and 10 in all animals and increased progressively until the animals died or met the criteria for euthanasia. Virus was detected in all organs tested, namely adrenal glands, spleen, liver, duodenum, jejunum, colon, bone marrow, lymph nodes, thymus, heart, lungs, pleural fluid, kidneys, skeletal muscle, pancreas, salivary glands, ovaries, bladder, cerebrum, cerebellum, brain stem, cerebrospinal fluid, and aqueous humor of the eye. The largest quantities of virus were generally detected in adrenal glands, spleen, liver, bone marrow, and intestines. The microscopic lesions included mononuclear pulmonary arteritis with intraluminal 
mononuclear cell aggregates and mononuclear cell infiltration of the subendothelium and the pulmonary arterial wall accompanied by endothelial hypertrophy and hyperplasia, hepatocellular necrosis, interstitial pneumonia, adrenal gland necrosis, encephalitis and uveitis. Therefore, infected rhesus monkeys exhibited pathologic lesions similar to human Lassa fever, such as the amounts and organ distribution of virus, necrosis of hepatocytes, adrenal cortical cells, and splenic marginal zone of the periaortic lymphocytic sheath, and interstitial nephritis. However, meningoencephalomeningitis, pulmonary vasculitis, systemic arteritis, and skeletal myositis were significantly more prominent in the monkey model than in human Lassa fever [92,96]. The clinical manifestations of LASV-infected cynomolgus macaques included fever, weight loss, depression, and acute respiratory syndrome. Other clinical features included thrombocytopenia, lymphopenia, lymphadenopathy, splenomegaly, infiltration of mononuclear cells, and pathologic alterations in the liver, lungs, and endothelium, which essentially mirrored observations from human cases [83]. An additional feature of the disease observed in cynomolgus monkeys was multifocal to severe central nervous system lesions at terminal stages. Dysregulation of the host immune response characterized by increased circulating levels of proinflammatory cytokines/chemokines including IL-1 $\beta$, IL-6, MCP-1, and eotaxin were detected in the infected animals. Histopathologic evaluation of tissues revealed a sequence of events in LASV infection in cynomolgus macaques, which is initiated with dendritic cells in the lymphoid tissues, then progresses to infection of Kupffer cells in liver and parenchymal cells in liver and adrenal glands, and endothelial cells in various organs including the central nervous system [95]. Experimental infection of common marmosets results in systemic disease from clinical and pathologic standpoints highly similar to disease observed in fatal cases of Lassa fever in humans. Among the main clinical features are fever, weight loss, high viremia and viral RNA loads in tissues, elevated levels of liver enzymes, and severe morbidity between days 15 and 20. Histopathologic changes include multifocal hepatic necrosis associated with mild inflammation and hepatocyte proliferation, adrenal necrosis, lymphoid depletion, and interstitial nephritis. The necrotic hepatocellular foci observed in LASV-infected marmosets contained predominantly macrophages with the near absence of CD20-, CD8-, or CD3-positive lymphocytes, markedly decreased expression of MHC-II molecules, and hepatocyte proliferation. The levels of MHC-II expression were also significantly reduced in lymph nodes. Overall numbers of CD20- and CD3-positive lymphocytes in the spleen of infected animals were reduced, and the destruction of lymphoid follicular architecture was evident [94]. These findings strongly suggest that the replication of LASV in target tissues may cause pathologic changes that directly impair the adaptive immune response to LASV infection.

\section{Acknowledgments}

N. E. Yun was supported by funding from the Institute for Human Infections and Immunity, University of Texas Medical Branch.

\section{Conflict of Interest}

The authors declare no conflict of interest". 


\section{References and Notes}

1. Wulff, H.; Lange, J.V.; Webb, P.A. Interrelationships among arenaviruses measured by indirect immunofluorescence. Intervirology 1978, 9, 344-350.

2. Bowen, M.D.; Peters, C.J.; Nichol, S.T. Phylogenetic analysis of the Arenaviridae: patterns of virus evolution and evidence for cospeciation between arenaviruses and their rodent hosts. Mol. Phylogenet. Evol. 1997, 8, 301-316.

3. Salazar-Bravo, J.; Ruedas, L.A.; Yates, T.L. Mammalian reservoirs of arenaviruses. Curr. Top. Microbiol. Immunol. 2002, 262, 25-63.

4. McCormick, J.B.; Webb, P.A.; Krebs, J.W.; Johnson, K.M.; Smith, E.S. A prospective study of the epidemiology and ecology of Lassa fever. J. Infect. Dis. 1987, 155, 437-444.

5. Gonzalez, J.P.J.; Bowen, M.D.; Nichol, S.T.; Rico-Hesse, R. Genetic characterization and phylogeny of Sabiá virus, an emergent pathogen in Brazil. Virology 1996, 221, 318-324.

6. Peters, C.J., Michael Buchmeier, Pierre E. Rollin, and Thomas G. Ksiazek Arenaviruses. In Fields Virology; Third edition ed.; Bernard N. Fields, D. M. K., Peter M. Howley, Ed. Lippincott-Raven Publishers: Philadelphia, 1996; Vol. Volume 2, pp. 1521-1551.

7. Briese, T.; Paweska, J.T.; McMullan, L.K.; Hutchison, S.K.; Street, C.; Palacios, G.; Khristova, M.L.; Weyer, J.; Swanepoel, R.; Egholm, M.; Nichol, S.T.; Lipkin, W.I. Genetic detection and characterization of Lujo virus, a new hemorrhagic fever-associated arenavirus from southern Africa. PLoS Pathog. 2009, 5, e1000455.

8. Delgado, S.; Erickson, B.R.; Agudo, R.; Blair, P.J.; Vallejo, E.; Albariño, C.G.; Vargas, J.; Comer, J.A.; Rollin, P.E.; Ksiazek, T.G.; Olson, J.G.; Nichol, S.T. Chapare virus, a newly discovered arenavirus isolated from a fatal hemorrhagic fever case in Bolivia. PLoS Pathog. 2008, 4, e1000047.

9. Salvato, M.S.; Clegg, J.C.S.; Buchmeier, M.J.; Charrel, R.N.; Gonzales, J.P.; Lukashevich, I.S.; Peters, C.J.; Rico-Hesse, R.; Romanowski, V. Family Arenaviridae. In Virus Taxonomy: Ninth Report of the International Committee on Taxonomy of Viruses; King, A. M. Q.; Adams, M. J.; Carstens, E. B.; Lefkowitz, E. F., Eds.; Academic Press: 2012; pp. 714-723.

10. Buchmeier, M.J., Peters, C.J., and de la Torre, J.C. Arenaviridae: The viruses and their replication. In Fields Virology, Fift Edition; Knipe, D. M.; Holey, P.M., Eds. 2007; Vol. 2, pp. 1792-1827.

11. Kranzusch, P.J.; Schenk, A.D.; Rahmeh, A.A.; Radoshitzky, S.R.; Bavari, S.; Walz, T.; Whelan, S.P.J. Assembly of a functional Machupo virus polymerase complex. Proceedings of the National Academy of Sciences 2010, 107, 20069-20074.

12. Salvato, M.S.; Shimomaye, E.M. The completed sequence of lymphocytic choriomeningitis virus reveals a unique RNA structure and a gene for a zinc finger protein. Virology 1989, 173, 1-10.

13. Franze-Fernandez, M.-T.; Zetina, C.; Iapalucci, S.; Lucero, M.A.; Bouissou, C.; Lopez, R.; Rey, O.; Daheli, M.; Cohen, G.N.; Zakin, M.M. Molecular structure and early events in the replication of Tacaribe arenavirus S RNA. Virus Res. 1987, 7, 309-324.

14. Meyer, B.J.; Southern, P.J. Concurrent sequence analysis of $5^{\prime}$ and $3^{\prime}$ RNA termini by intramolecular circularization reveals $5^{\prime}$ nontemplated bases and $3^{\prime}$ terminal heterogeneity for lymphocytic choriomeningitis virus mRNAs. J. Virol. 1993, 67, 2621-2627. 
15. Qi, X.; Lan, S.; Wang, W.; Schelde, L.M.; Dong, H.; Wallat, G.D.; Ly, H.; Liang, Y.; Dong, C. Cap binding and immune evasion revealed by Lassa nucleoprotein structure. Nature 2010, 468, 779-783.

16. Lelke, M.; Brunotte, L.; Busch, C.; Günther, S. An N-terminal region of Lassa virus L protein plays a critical role in transcription but not replication of the virus genome. J. Virol. 2010, 84, 1934-1944.

17. Morin, B.; Coutard, B.; Lelke, M.; Ferron, F.; Kerber, R.; Jamal, S.; Frangeul, A.; Baronti, C.; Charrel, R.; de Lamballerie, X.; Vonrhein, C.; Lescar, J.; Bricogne, G.; Günther, S.; Canard, B. The N-terminal domain of the arenavirus L protein is an RNA endonuclease essential in mRNA transcription. PLoS Pathog. 2010, 6, e1001038.

18. Garcin, D.; Kolakofsky, D. A novel mechanism for the initiation of Tacaribe arenavirus genome replication. J. Virol. 1990, 64, 6196-6203.

19. Garcin, D.; Kolakofsky, D. Tacaribe arenavirus RNA synthesis in vitro is primer dependent and suggests an unusual model for the initiation of genome replication. J. Virol. 1992, 66, 1370-1376.

20. Marq, J.-B.; Kolakofsky, D.; Garcin, D. Unpaired 5' ppp-nucleotides, as found in arenavirus double-stranded RNA panhandles, are not recognized by RIG-I. J. Biol.Chem. 2010, 285, 1820818216.

21. Cornu, T.I.; de la Torre, J.C. RING finger Z protein of lymphocytic choriomeningitis virus (LCMV) inhibits transcription and RNA replication of an LCMV S-segment minigenome. J. Virol. 2001, 75, 9415-9426.

22. Cornu, T.I.; de la Torre, J.C. Characterization of the arenavirus RING finger $Z$ protein regions required for Z-mediated inhibition of viral RNA synthesis. J. Virol. 2002, 76, 6678-6688.

23. Cornu, T.I.; Feldmann, H.; de la Torre, J.C. Cells expressing the RING finger Z protein are resistant to arenavirus infection. J. Virol. 2004, 78, 2979-2983.

24. Lopez, N.; Jacamo, R.; Franze-Fernandez, M.T. Transcription and RNA replication of Tacaribe virus genome and antigenome analogs require $\mathrm{N}$ and $\mathrm{L}$ proteins: $\mathrm{Z}$ protein is an inhibitor of these processes. J. Virol. 2001, 75, 12241-12251.

25. Hass, M.; Golnitz, U.; Muller, S.; Becker-Ziaja, B.; Gunther, S. Replicon system for Lassa virus. J. Virol. 2004, 78, 13793-13803.

26. Martinez-Sobrido, L.; Zuniga, E.I.; Rosario, D.; Garcia-Sastre, A.; de la Torre, J.C. Inhibition of the type I interferon response by the nucleoprotein of the prototypic arenavirus lymphocytic choriomeningitis virus. J. Virol. 2006, 80, 9192-9199.

27. Martinez-Sobrido, L.; Giannakas, P.; Cubitt, B.; Garcia-Sastre, A.; de la Torre, J.C. Differential inhibition of type I interferon induction by arenavirus nucleoproteins. J. Virol. 2007, 81, 1269612703.

28. Martínez-Sobrido, L.; Emonet, S.; Giannakas, P.; Cubitt, B.; García-Sastre, A.; de la Torre, J.C. Identification of amino acid residues critical for the anti-interferon activity of the nucleoprotein of the prototypic arenavirus lymphocytic choriomeningitis virus. J. Virol. 2009, 83, 11330-11340.

29. Eckerle, L.D.; Becker, M.M.; Halpin, R.A.; Li, K.; Venter, E.; Lu, X.; Scherbakova, S.; Graham, R.L.; Baric, R.S.; Stockwell, T.B.; Spiro, D.J.; Denison, M.R. Infidelity of SARS-CoV Nsp14exonuclease mutant virus replication is revealed by complete genome sequencing. PLoS Pathog. 2010, 6, e1000896. 
30. Borden, K.L.; Campbell Dwyer, E.J.; Salvato, M.S. An arenavirus RING (zinc-binding) protein binds the oncoprotein promyelocyte leukemia protein (PML) and relocates PML nuclear bodies to the cytoplasm. J. Virol. 1998, 72, 758-766.

31. Campbell Dwyer, E.J.; Lai, H.; MacDonald, R.C.; Salvato, M.S.; Borden, K.L. The lymphocytic choriomeningitis virus RING protein $\mathrm{Z}$ associates with eukaryotic initiation factor $4 \mathrm{E}$ and selectively represses translation in a RING-dependent manner. J. Virol. 2000, 74, 3293-3300.

32. Djavani, M.; Rodas, J.; Lukashevich, I.S.; Horejsh, D.; Pandolfi, P.P.; Borden, K.L.; Salvato, M.S. Role of the promyelocytic leukemia protein PML in the interferon sensitivity of lymphocytic choriomeningitis virus. J. Virol. 2001, 75, 6204-6208.

33. Volpon, L.; Osborne, M.J.; Capul, A.A.; de la Torre, J.C.; Borden, K.L.B. Structural characterization of the Z RING-eIF4E complex reveals a distinct mode of control for eIF4E. Proceedings of the National Academy of Sciences 2010, 107, 5441-5446.

34. Frame, J.D.; Baldwin, J.M.; Gocke, D.J.; Troup, J.M. Lassa fever, a new virus disease of man from West Africa. Am. J. Trop. Med. Hyg. 1970, 19, 670-676.

35. Buckley, S.M.; Casals, J. Lassa fever, a new virus disease of man from West Africa. 3. Isolation and characterization of the virus. Am. J. Trop. Med. Hyg. 1970, 19, 680-691.

36. Monath, T.P.; Maher, M.; Casals, J.; Kissling, R.E.; Cacciapuoti, A. Lassa fever in the Eastern Province of Sierra Leone, 1970-1972. II. Clinical observations and virological studies on selected hospital cases. Am. J. Trop. Med. Hyg. 1974, 23, 1140-1149.

37. Lukashevich, I.S.; Clegg, J.C.S.; Sidibe, K. Lassa virus activity in Guinea: Distribution of human antiviral antibody defined using enzyme-linked immunosorbent assay with recombinant antigen. J.Med. Virol. 1993, 40, 210-217.

38. Meulen, J.T.; Lukashevich, I.; Sidibe, K.; Inapogui, A.; Marx, M.; Dorlemann, A.; Yansane, M.L.; Koulemou, K.; Chang-Claude, J.; Schmitz, H. Hunting of peridomestic rodents and consumption of their meat as possible risk factors for rodent-to-human transmission of Lassa virus in the Republic of Guinea. Am. J. Trop. Med. Hyg. 1996, 55, 661-666.

39. Frame, J.D.; Yalley-Ogunro, J.E.; Hanson, A.P. Endemic Lassa fever in Liberia. V. Distribution of Lassa virus activity in Liberia: hospital staff surveys. Trans. R. Soc. Trop. Med. Hyg. 1984, 78, 761-763.

40. Monath, T.P.; Mertens, P.E.; Patton, R.; Moser, C.R.; Baum, J.J.; Pinneo, L.; Gary, G.W.; Kissling, R.E. A hospital epidemic of Lassa fever in Zorzor, Liberia, March-April 1972. Am. J. Trop. Med. Hyg. 1973, 22, 773-779.

41. Monson, M.H.; Frame, J.D.; Jahrling, P.B.; Alexander, K. Endemic Lassa fever in Liberia. I. Clinical and epidemiological aspects at Curran Lutheran Hospital, Zorzor, Liberia. Trans. R. Soc. Trop. Med. Hyg. 1984, 78, 549-553.

42. Carey, D.E.; Kemp, G.E.; White, H.A.; Pinneo, L.; Addy, R.F.; Fom, A.L.M.D.; Stroh, G.; Casals, J.; Henderson, B.E. Lassa fever: Epidemiological aspects of the 1970 epidemic, Jos, Nigeria. Trans. R. Soc. Trop. Med. Hyg. 1972, 66, 402-408.

43. Tomori, O.; Fabiyi, A.; Sorungbe, A.; Smith, A.; McCormick, J.B. Viral hemorrhagic fever antibodies in Nigerian populations. Am. J. Trop. Med. Hyg. 1988, 38, 407-410.

44. White, H.A. Lassa fever. A study of 23 hospital cases. Trans. R. Soc. Trop. Med. Hyg. 1972, 66, 390-401. 
45. Troup, J.M.; White, H.A.; Fom, A.L.M.D.; Carey, D.E. An outbreak of Lassa fever on the Jos Plateau, Nigeria, in January-February 1970. Am. J. Trop. Med. Hyg. 1970, 19, 695-696.

46. Frame, J.D. Surveillance of Lassa fever in missionaries stationed in West Africa. Bull. World Health Organ. 1975, 52, 593-598.

47. Günther, S.; Emmerich, P.; Laue, T.; Kühle, O.; Asper, M.; Jung, A.; Grewing, T.; ter Meulen, J.; Schmitz, H. Imported Lassa fever in Germany: molecular characterization of a new Lassa virus strain. Emerg. Infect. Dis. 2000, 6, 466-476.

48. Gonzalez, J.P.; Emonet, S.; Lamballerie, X.D.; Charrel, R. Arenaviruses. In Wildlife and Emerging Zoonotic Diseases: The Biology, Circumstances and Consequences of Cross-Species Transmission; Childs, J. E.; Mackenzie, J. S.; Richt, J. A., Eds.; Springer Berlin Heidelberg: 2007; Vol. 315, pp. 253-288.

49. Safronetz, D.; Lopez, J.E.; Sogoba, N.; Traore', S.F.; Raffel, S.J.; Fischer, E.R.; Ebihara, H.; Branco, L.; Garry, R.F.; Schwan, T.G.; Feldmann, H. Detection of Lassa virus, Mali. Emerg. Infect. Dis. 2010, 16, 1123-1126.

50. Lassa Fever Fact Sheet. Centers for Disease Control and Prevention. Available online: http://www.cdc.gov/ncidod/dvrd/spb/mnpages/dispages/Fact_Sheets/Lassa_Fever_Fact_Sheet.pdf

51. Keenlyside, R.A.; McCormick, J.B.; Webb, P.A.; Smith, E.; Elliott, L.; Johnson, K.M. Casecontrol study of Mastomys natalensis and humans in Lassa virus-infected households in Sierra Leone. Am. J. Trop. Med. Hyg. 1983, 32, 829-837.

52. Fisher-Hoch, S.P.; Tomori, O.; Nasidi, A.; Perez-Oronoz, G.I.; Fakile, Y.; Hutwagner, L.; McCormick, J.B. Review of cases of nosocomial Lassa fever in Nigeria: the high price of poor medical practice. BMJ 1995, 311, 857-859.

53. Helmick, C.G.; Webb, P.A.; Scribner, C.L.; Krebs, J.W.; McCormick, J.B. No evidence for increased risk of Lassa fever infection in hospital staff. Lancet 1986, 2, 1202-1205.

54. Stephenson, E.H.; Larson, E.W.; Dominik, J.W. Effect of environmental factors on aerosolinduced lassa virus infection. J. Med. Virol. 1984, 14, 295-303.

55. Peters, C.J.; Jahrling, P.B.; Liu, C.T.; Kenyon, R.H.; McKee, K.T., Jr.; Barrera Oro, J.G. Experimental studies of arenaviral hemorrhagic fevers. Curr. Top. Microbiol. Immunol. 1987, 134, 5-68.

56. Monath, T.P.; Newhouse, V.F.; Kemp, G.E.; Setzer, H.W.; Cacciapuoti, A. Lassa virus isolation from Mastomys natalensis rodents during an epidemic in Sierra Leone. Science 1974, 185, 263265.

57. Walker, D.H.; Wulff, H.; Lange, J.V.; Murphy, F.A. Comparative pathology of Lassa virus infection in monkeys, guinea pigs, and Mastomys natalensis. Bull. World Health Organ. 1975, 52, 523-535.

58. McCormick, J.B.; King, I.J.; Webb, P.A.; Johnson, K.M.; O'Sullivan, R.; Smith, E.S.; Trippel, S.; Tong, T.C. A case-control study of the clinical diagnosis and course of Lassa fever. J. Infect. Dis. 1987, 155, 445-455.

59. Walker, D.H.; McCormick, J.B.; Johnson, K.M.; Webb, P.A.; Komba-Kono, G.; Elliott, L.H.; Gardner, J.J. Pathologic and virologic study of fatal Lassa fever in man. Am. J. Pathol. 1982, 107, 349-356. 
60. Cummins D, M.J.B.B.D.; et al. Acute sensorineural deafness in Lassa fever. JAMA-J. Am. Med. Assoc. 1990, 264, 2093-2096.

61. Johnson, K.M.; McCormick, J.B.; Webb, P.A.; Smith, E.S.; Elliott, L.H.; King, I.J. Clinical virology of Lassa fever in hospitalized patients. J. Infect. Dis. 1987, 155, 456-464.

62. Demby, A.H.; Chamberlain, J.; Brown, D.W.; Clegg, C.S. Early diagnosis of Lassa fever by reverse transcription-PCR. J.Clin. Microbiol. 1994, 32, 2898-2903.

63. Trappier, S.G.; Conaty, A.L.; Farrar, B.B.; Auperin, D.D.; McCormick, J.B.; Fisher-Hoch, S.P. Evaluation of the polymerase chain reaction for diagnosis of Lassa virus infection. Am. J. Trop. Med. Hyg. 1993, 49, 214-221.

64. Flatz, L.; Rieger, T.; Merkler, D.; Bergthaler, A.; Regen, T.; Schedensack, M.; Bestmann, L.; Verschoor, A.; Kreutzfeldt, M.; Brück, W.; Hanisch, U.-K.; Günther, S.; Pinschewer, D.D. T celldependence of Lassa fever pathogenesis. PLoS Pathog. 2010, 6, e1000836.

65. Edington, G.M.; White, H.A. The pathology of Lassa fever: A tribute to the late Dr. J.M. Troup. Trans. R. Soc. Trop. Med. Hyg. 1972, 66, 381-389.

66. McCormick, J.B.; Walker, D.H.; King, I.J.; Webb, P.A.; Elliott, L.H.; Whitfield, S.G.; Johnson, K.M. Lassa virus hepatitis: a study of fatal Lassa fever in humans. Am. J. Trop. Med. Hyg. 1986, $35,401-407$.

67. Winn, W.C.; Walker, D.H. The pathology of human Lassa fever. Bull. World Health Organ. 1975, 52, 535-545.

68. Fisher-Hoch, S.; McCormick, J.B.; Sasso, D.; Craven, R.B. Hematologic dysfunction in Lassa fever. J. Med. Virol. 1988, 26, 127-135.

69. Schmitz, H.; Kohler, B.; Laue, T.; Drosten, C.; Veldkamp, P.J.; Gunther, S.; Emmerich, P.; Geisen, H.P.; Fleischer, K.; Beersma, M.F.; Hoerauf, A. Monitoring of clinical and laboratory data in two cases of imported Lassa fever. Microbes Infect. 2002, 4, 43-50.

70. Mahanty, S.; Bausch, D.G.; Thomas, R.L.; Goba, A.; Bah, A.; Peters, C.J.; Rollin, P.E. Low levels of interleukin-8 and interferon-inducible protein-10 in serum are associated with fatal infections in acute Lassa fever. J. Infect. Dis. 2001, 183, 1713-1721.

71. Lukashevich, I.S.; Maryankova, R.; Vladyko, A.S.; Nashkevich, N.; Koleda, S.; Djavani, M.; Horejsh, D.; Voitenok, N.N.; Salvato, M.S. Lassa and Mopeia virus replication in human monocytes/macrophages and in endothelial cells: Different effects on IL-8 and TNF- $\alpha$ gene expression. J. Med. Virol. 1999, 59, 552-560.

72. Mahanty, S.; Hutchinson, K.; Agarwal, S.; McRae, M.; Rollin, P.E.; Pulendran, B. Cutting edge: impairment of dendritic cells and adaptive immunity by Ebola and Lassa viruses. J. Immunol. 2003, 170, 2797-2801.

73. Baize, S.; Kaplon, J.; Faure, C.; Pannetier, D.; Georges-Courbot, M.C.; Deubel, V. Lassa virus infection of human dendritic cells and macrophages is productive but fails to activate cells. $J$. Immunol. 2004, 172, 2861-2869.

74. Pannetier, D.; Reynard, S.; Russier, M.; Journeaux, A.; Tordo, N.; Deubel, V.; Baize, S. Human dendritic cells infected with the nonpathogenic Mopeia virus induce stronger T-cell responses than those infected with Lassa virus. J. Virol. 2011, 85, 8293-8306. 
75. Jahrling, P.B.; Frame, J.D.; Rhoderick, J.B.; Monson, M.H. Endemic Lassa fever in Liberia. IV. Selection of optimally effective plasma for treatment by passive immunization. Trans. R. Soc. Trop. Med. Hyg. 1985, 79, 380-384.

76. ter Meulen, J.; Koulemou, K.; Wittekindt, T.; Windisch, K.; Strigl, S.; Conde, S.; Schmitz, H. Detection of Lassa virus antinucleoprotein immunoglobulin $\mathrm{G}(\mathrm{IgG})$ and $\operatorname{IgM}$ antibodies by a simple recombinant immunoblot assay for field use. J. Clin. Microbiol. 1998, 36, 3143-3148.

77. Hummel, K.B.; Martin, M.L.; Auperin, D.D. Baculovirus expression of the glycoprotein gene of Lassa virus and characterization of the recombinant protein. Virus Res. 1992, 25, 79-90.

78. Lloyd, G.; Barber, G.N.; Clegg, J.C.S.; Kelly, P. Identification of Lassa fever virus infection with recombinant nucleocapsid protein antigen. Lancet 1989, 334, 1222.

79. Günther, S.; Kühle, O.; Rehder, D.; Odaibo, G.; Olaleye, D.; Emmerich, P.; ter Meulen, J.; Schmitz, H. Antibodies to Lassa virus Z protein and nucleoprotein co-occur in human sera from Lassa fever endemic regions. Med. Microbiol. Immun. 2001, 189, 225-229.

80. Ruo, S.L.; Mitchell, S.W.; Kiley, M.P.; Roumillat, L.F.; Fisher-Hoch, S.P.; McCormick, J.B. Antigenic relatedness between arenaviruses defined at the epitope level by monoclonal antibodies. J. Gen. Virol. 1991, 72, 549-555.

81. Hufert, F.T.; Lüdke, W.; Schmitz, H. Epitope mapping of the Lassa virus nucleoprotein using monoclonal anti-nucleocapsid antibodies. Arch. Virol. 1989, 106, 201-212.

82. Vladyko, A.S.; I., B.S.; N., L.N.; Lukashevich, I.S. Characteristics of monoclonal antibodies against Lassa virus. Mol. Gen. Mikrobiol. Virusol. 1987, 37-40.

83. Baize, S.; Marianneau, P.; Loth, P.; Reynard, S.; Journeaux, A.; Chevallier, M.; Tordo, N.; Deubel, V.; Contamin, H. Early and strong immune responses are associated with control of viral replication and recovery in Lassa virus-infected cynomolgus monkeys. J. Virol. 2009, 83, 58905903.

84. ter Meulen, J.; Badusche, M.; Kuhnt, K.; Doetze, A.; Satoguina, J.; Marti, T.; Loeliger, C.; Koulemou, K.; Koivogui, L.; Schmitz, H.; Fleischer, B.; Hoerauf, A. Characterization of human CD4+ T-cell clones recognizing conserved and variable epitopes of the Lassa virus nucleoprotein. J. Virol. 2000, 74, 2186-2192.

85. Meulen, J.T.; Badusche, M.; Satoguina, J.; Strecker, T.; Lenz, O.; Loeliger, C.; Sakho, M.; Koulemou, K.; Koivogui, L.; Hoerauf, A. Old and New World arenaviruses share a highly conserved epitope in the fusion domain of the glycoprotein 2, which is recognized by Lassa virusspecific human CD4+ T-cell clones. Virology 2004, 321, 134-143.

86. Lukashevich, I.S. Lassa virus lethality for inbred mice. Ann. Soc. Belg. Med. Trop. 1985, 65, $207-$ 209.

87. Oldstone, M.B. Biology and pathogenesis of lymphocytic choriomeningitis virus infection. In Arenaviruses; Oldstone, M. B., Ed. 2002; Vol. 263, pp. 83-118.

88. Jahrling, P.B.; Smith, S.; Hesse, R.A.; Rhoderick, J.B. Pathogenesis of Lassa virus infection in guinea pigs. Infect. Immun. 1982, 37, 771-778.

89. Jahrling, P.B.; Frame, J.D.; Smith, S.B.; Monson, M.H. Endemic Lassa fever in Liberia. III. Characterization of Lassa virus isolates. Trans. R. Soc. Trop. Med. Hyg. 1985, 79, 374-379.

90. Walker, D.H. Pathology and pathogenesis of arenavirus infections. Curr. Top. Microbiol. Immunol. 1987, 133, 89-113. 
91. Walker, D.H.; Wulff, H.; Murphy, F.A. Experimental Lassa virus infection in the squirrel monkey. Am. J. Pathol. 1975, 80, 261-278.

92. Walker, D.H.; Johnson, K.M.; Lange, J.V.; Gardner, J.J.; Kiley, M.P.; McCormick, J.B. Experimental infection of rhesus monkeys with Lassa virus and a closely related arenavirus, Mozambique virus. J. Infect. Dis. 1982, 146, 360-368.

93. Evseev, A.A.; Dvoretskaia, V.I.; Bogatikov, G.V.; Phenichnov, V.A.; Mustafin, R.M. Experimental Lassa fever in hamadryas baboons. Vopr. Virusol. 1991, 36, 150-152.

94. Carrion, R., Jr.; Brasky, K.; Mansfield, K.; Johnson, C.; Gonzales, M.; Ticer, A.; Lukashevich, I.; Tardif, S.; Patterson, J., Lassa virus infection in experimentally infected marmosets: liver pathology and immunophenotypic alterations in target tissues. In 2007; Vol. 81, pp 6482-6490.

95. Hensley, L.; Smith, M.; Geisbert, J.; Fritz, E.; Daddario-DiCaprio, K.; Larsen, T.; Geisbert, T. Pathogenesis of Lassa fever in cynomolgus macaques. Virol. J. 2011, 8, 205.

96. Lange, J.V.; Mitchell, S.W.; McCormick, J.B.; Walker, D.H.; Evatt, B.L.; Ramsey, R.R. Kinetic study of platelets and fibrinogen in Lassa virus-infected monkeys and early pathologic events in Mopeia virus-infected monkeys. Am. J. Trop. Med. Hyg. 1985, 34, 999-1007.

(C) 2012 by the authors; licensee MDPI, Basel, Switzerland. This article is an open access article distributed under the terms and conditions of the Creative Commons Attribution license (http://creativecommons.org/licenses/by/3.0/). 